\section{Case Report}

Volume : 1 , Issue :1

April 2017

Pages: $11-15$

\title{
Ultrasonographic findings in two dogs with canine parvoviral enteritis
}

\section{Burçak Özkan*}

\begin{abstract}
Canine parvoviral enteritis (CPV) is one of the most common diseases why puppies are presented to clinicians. Being non-enveloped, single striated DNA viruses, parvoviruses cause disease in a variety of wild and domestic mammalians. Three subtypes of the virus have similar pathogenicity resulting in clinical disease. In spite of affecting also older animals, the disease most often strikes in pups. Cardiac and intestinal forms are two types of the disease. CPV is highly contagious and severe but healed dogs gain lifetime immunity. Abdominal ultrasonography (USG) has greater accuracy since it is a non-invasive but efficient method. Thus, it may be helpful to diagnose the disease when accompanied by clinical and laboratory tests. The aim of this case report is to discuss USG findings, clinical symptoms and healing procedure of two dogs suffering from CPV represented with complaints of hemorrhagic diarrhoea, vomitus and lack of appetite and water intake as well as progressive weight loss. Non-specific signs were found in both dogs. One puppy was infested with ascariasis. Routine treatment was adopted for both puppies. Definitive diagnosis is established with the help of anamnesis, clinical symptoms and laboratory tests. USG is a non-invasive, fast and reliable method and may be employed as a supportive examination for enteritis detection in order to exclude other intestinal diseases necessitating laparotomy. In our case, intestinal USG was performed with a $5-7.5 \mathrm{mHz}$ transducer. Altered intestinal wall structure and damaged intestinal individualization were noted. Both patients were healed.
\end{abstract}

Keywords: dog, canine parvovirus, ultrasonography

DOI: 10.30704/http-www-jivs-net.295259

To cite this article: Özkan, B. (2017). Ultrasonographic findings in two dogs with canine parvoviral enteritis. Journal of Istanbul Veterinary Sciences, 1(1), 11-15.

Abbreviated Title: J Ist Vet Sci

\section{Introduction}

Parvoviruses are very small, non-enveloped, singlestriated DNA viruses that replicate in rapidly dividing cells and they cause diseases in a variety of wild and domestic mammalians (Goddard and Leisewitz, 2010). Canine parvovirus-2 (CPV-2) emerged as a cause of acute canine enteritis in the last quarter of 1970 possibly from another carnivore parvovirus, spread rapidly worldwide (Decaro and Buonavoglia, 2012). In the first quarter of 1980s CPV-2 evolved into two subtypes (CPV$2 \mathrm{a}$ and CPV-2b), while in 2000 a third subtype (CPV-2c) was documented (Ntafis et al., 2010). Three subtypes of the virus are thought to have similar pathogenicity leading to indiscernible clinical diseases (Marcovich et al., 2012). These subtypes are the causative agents of acute hemorrhagic enteritis and myocarditis in dogs. Since its emergence in 1978, canine parvoviral enteritis was an important cause of morbidity and mortality in

*Corresponding Author: Burçak Özkan

E mail: burcakozkan1974@yahoo.com young dogs (Kumar and Nandi, 2010). The disease most often strikes in pups between 6 and 20 weeks old but older animals are also affected. Still, the prevalence, morbidity and mortality of the disease is very common in many countries. Both direct contact with contaminated feces and indirect contact with contaminated fomites as well as insects and rodents cause the infection. The virus remains infectious in contaminated environment for months (Prittie, 2004). Two types of the disease are the cardiac and the intestinal forms. The first one only seen in those infected in utero is presented with respiratory and cardiac symptoms. The second one is highly contagious and severe. Due to its affinity, virus invades quickly mitotic intestinal cells, bone marrow and lymph nodes after having caused intestinal impairment. In case an infected dog is healed, lifetime immunity is gained (Nwoha, 2011).

Canine parvovirus is commonly diagnosed by Journal homepage: www.jivs.net http://dergipark.gov.tr/http-www-jivs-net 
tests. Diagnostic tests include detection of CPV in the feces by ELISA, polymerase chain reaction (PCR) and necropsy with histopathology (Mathios et al., 2016). Apart from evaluation of clinical symptoms and laboratory tests, USG permits non-invasive examination of motility and thickness of intestinal tract in most clinical conditions. In spite of the rarity of such researches, the ultrasonographic examination of intestinal tract in infectious enteritis is established in dogs (Stander, 2009).

In human and animal studies, abdominal USG has greater accuracy and fewer equivocal results and it is preferred since it reduces the radiation exposure and costs (Sharma, 2010). Providing information of bowel wall thickness, layering of the wall, peristalsis, luminal contents and adjacent structures, ultrasound examination has become routine in intestinal disease investigation (Malancus, 2010). Advances in veterinary abdominal imaging enabled better investigation of the gastrointestinal tract and became an essential tool to examine small animal gastrointestinal diseases (Benigni, 2012). Normal gastrointestinal ultrasonographic appearance is well defined in dogs and cats (Mantis, 2008). The aim of this case report is to discuss the ultrasonographic findings and the clinical healing procedure of two dogs with CPV.

Table 1: Physiological and hematological parameters of the dogs on the first day of the treatment.

\begin{tabular}{lccc}
\hline $\begin{array}{l}\text { Physiological and } \\
\text { Hematological } \\
\text { Parameters }\end{array}$ & Dog-1 & Dog-2 & $\begin{array}{c}\text { Reference } \\
\text { Values }\end{array}$ \\
\hline $\begin{array}{l}\text { Temperature }\left({ }^{\circ} \mathrm{C}\right) \\
\text { Heart Rate }(B e a t / m i n)\end{array}$ & 35.8 & 36.1 & $37.5-39.2$ \\
Hematocrit $(\%)$ & 19 & 187 & $60-180$ \\
WBC $\left(\times 10^{3} / \mu \mathrm{L}\right)$ & 1.8 & 2.3 & $37-55$ \\
Albumin $(\mathrm{g} / \mathrm{dL})$ & 1.9 & - & $2.6-4.3$ \\
Glucose $(\mathrm{mg} / \mathrm{dL})$ & - & 50 & $60-125$ \\
\hline
\end{tabular}

\section{Case Presentation}

Two dogs with 3 and 4 months-old of age were presented with complaints of hemorrhagic diarrhoea, vomiting, lack of appetite and water intake and progressive weight loss. The first one was a mix breed female dog weighing $8 \mathrm{~kg}$ and the second dog was a 4 months-old German Shepherd weighing $10 \mathrm{~kg}$. The female was brought to the clinic 2 days after the problems began and the other dog on the first day. On the anamnesis, the owners said the dogs were vaccinated and protected against parasites. Both were indoor dogs and living without any other pets. Dogs were taken from a pet shop

\section{Clinical examination and findings}

Clinical examination revealed that both dogs were having similar nonspecific signs such as lethargy, decreased skin turgor, dehydration, cold extremities and enophtalmus with weight loss due to the lack of appetite and water intake accompanied by the pain detected on abdominal palpation. All signs were clearer on the female's examination. Yet a profuse hemorrhagic diarrhoea and vomitus was arousing attention in both. Haemogram and biochemical values of both puppies are presented in Table-1.

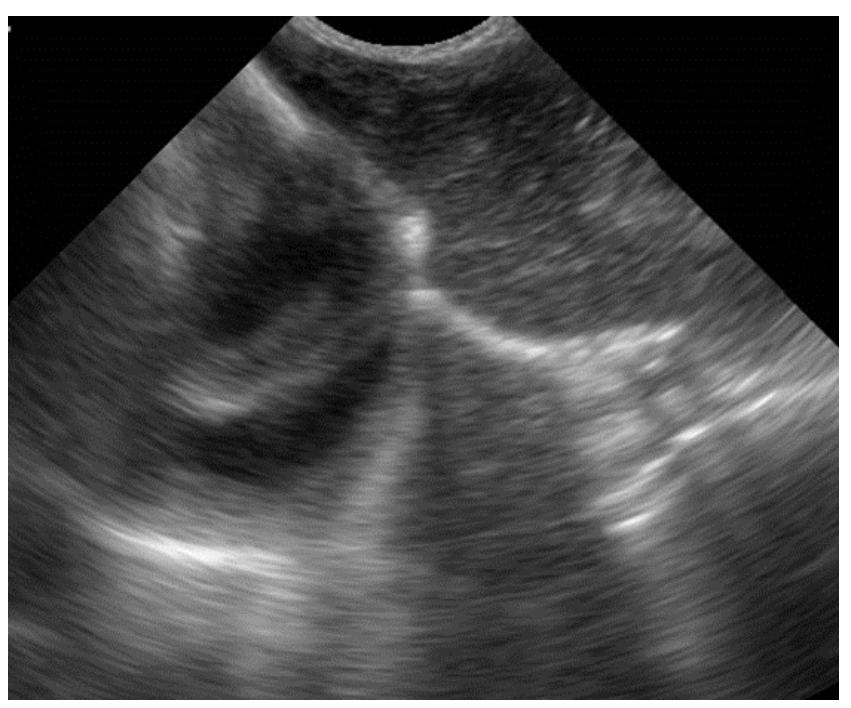

Figure 1. Normal ultrasonographic intestinal view in dog.

Gaita samples of both dogs were sent to a veterinary laboratory for parasitologic and microbiologic analyses and the examination revealed that the first patient was suffering from ascariasis (Toxocara canis). Body temperatures of both patients were normalized with the use of external heat and the cardiac frequencies reached normal values as the therapy went on. HCT value of Dog-1 was $26 \%$ after 12 hours of blood transfusion and the values were $35 \%$, and $38 \%$ for two dogs on day 7 . WBC values were $6.4 \times 10^{3} / \mu \mathrm{L}$ and 7.2 $\mathrm{x} 10^{3} / \mu \mathrm{L}$ respectively one week after the initial 
treatment. The same day Dog-1's albumin level was measured $2.7 \mathrm{~g} / \mathrm{dL}$ and Dog-2's glucose level $70 \mathrm{mg} / \mathrm{dL}$. Intestinal USG was performed for both puppies with a 5$7.5 \mathrm{mHz}$ transducer. The figures 2, 3, 4, 5 represent two dimensional B-Mode images of the ultrasonographic findings such as altered intestinal wall structure and damaged intestinal individualization.

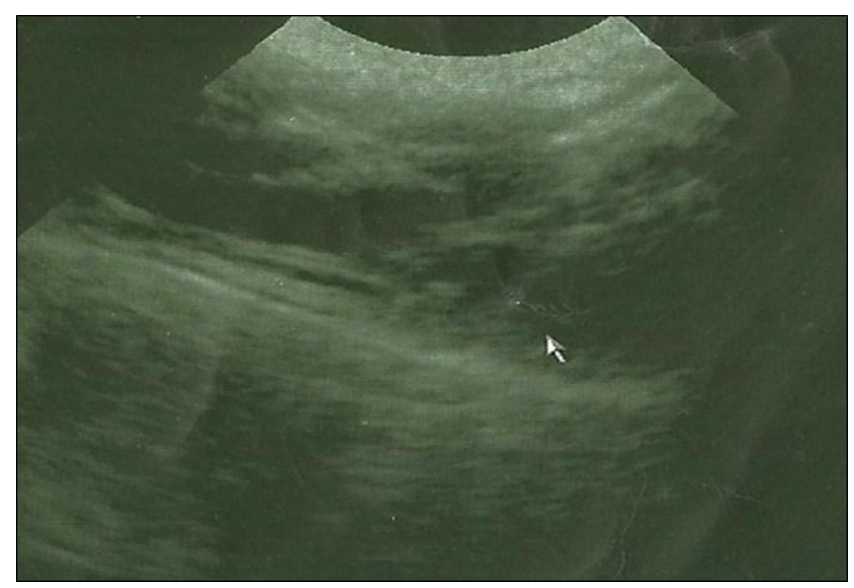

Figure 2. Dog-1. The thickening in intestinal wall and the intestinal loops with a low content as they lost their individualization. The striated structure is visible on the left side.

Diagnosis: Apart from the clinical and ultrasonographic examinations, hematologic, biochemical and laboratory analyses, confirmative diagnosis was made on a readily available positive faecal ELISA test for parvovirus to demonstrate CPV.

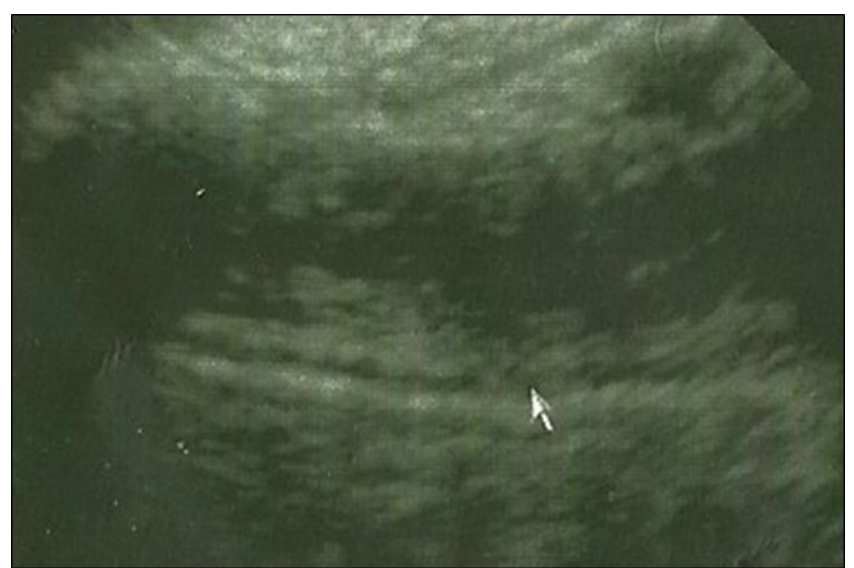

Figure 3. Dog-1. Longitudinal view of altered intestinal layers and thickened intestinal wall.

Treatment Adopted: Both puppies were hospitalized and kept isolated. Both food and water intake were withheld until vomiting ceased. As there was no hypovolemic shock but only dehydration and vomitus, Lactated Ringer's solution and $5 \%$ dextrose were chosen for fluid therapy for a week. During the first day, fluid was administered over 24 hours. Gradual oral alimentation started with Hills I/D prescription $\operatorname{diet}($ as vomiting ceased. Metoclopramide $\mathrm{HCl}$ (Metpamid®) $(1.0 \mathrm{mg} / \mathrm{kg} . \mathrm{IM} \mathrm{q} 8 \mathrm{~h}$ and then $0.5 \mathrm{mg} /$ kg.IM q8h.) was preferred as antiemetic and ranitidine (Ranitab®) $(2.0 \mathrm{mg} / \mathrm{kgSCq} 8 \mathrm{~h}$. then $1.0 \mathrm{mg} / \mathrm{kg} . q 12 \mathrm{~h}$. and continued with $0.5 \mathrm{mg} / \mathrm{kg} . \mathrm{q} 24 \mathrm{~h}$.) in order to protect gastric mucosa. Antimicrobial therapy was started with $\beta$-Lactam antibiotic (Ampicillin/ 22mg/ kg.IV/TID) (Ampisina $®$ ) and aminoglycoside (Gentamicin/ 4mg/kg.IV/TID) (Genta®) was added once the rehydration was established. The therapy was supported with Vitamine B complex (Bemiks Kompoze ®) Kompoze) (<15 kg.; 1-5ml.) Ascariasis was treated with pyrantel pamoate (Kontil@) (5mg/kg. PO) and in order to increase HCT value, blood transfusion was performed to the first patient.

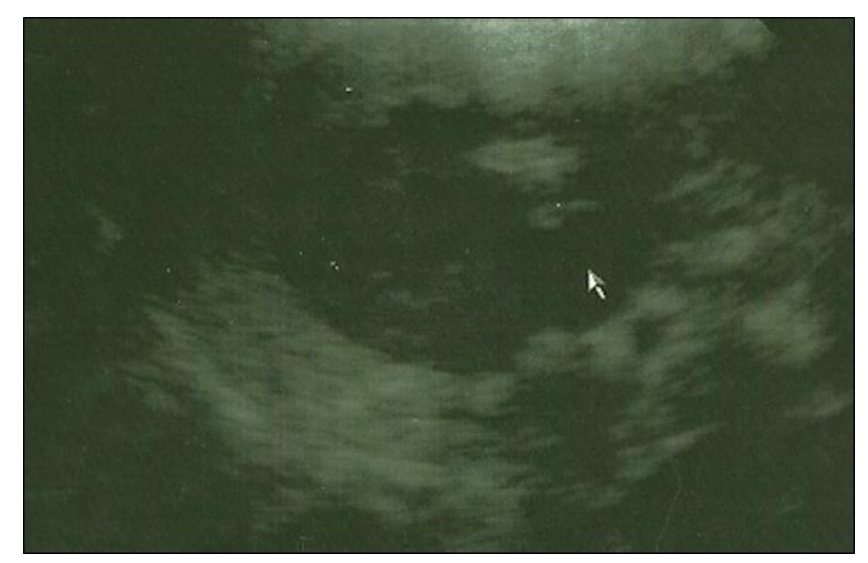

Figure 4. Dog 2. Longitudinal scan of small bowel segment distension with loss of wall layering due to the enteritis.

\section{Discussion}

Canine parvovirus may be a highly contagious disease of young dogs even when they are vaccinated. In addition some intestinal parasites form important cause of infectious enteritis (Nwoha, 2011). In our case, one of the patients was suffering from CPV and ascariasis was worsening the condition. Both dogs were taken from a pet shop and both vaccination and anti-parasitary procedure may have not been organized appropriately. The characteristic hemorrhagic diarrhoea results from villous damage, increased intestinal permeability and malassimilation due to abnormal mucosal function. Breakdown in the intestinal epithelial barrier causes intestinal bacterial translocation and bacterial endotoxin absorption into systemic circulation (Stander, 2009). 
Leukopenia and tachycardia are related to hemorrhage and anemia while low rectal temperature, cold extremities and abdominal pain were most consistent findings (Nwoha, 2011). Though they weren't confirmator, these symptoms and haemogram supported the diagnosis. Mild hypoalbuminemia in Dog -1 was related to gastrointestinal protein loss while the mild hypoglycemia in Dog-2 was accepted to be caused by malnutrition (Prittie, 2004).

Little information exists about ultrasonographic appearance in canine acute inflammatory intestinal diseases. Sonographic alterations related to CPV are fluid filled and atonic intestines, duodenal and jejunal mucosal layer thinning being or not accompanied by indistinct wall layers and irregular luminal mucosal surfaces, extensive duodenal and/or jejunal hyperecoic mucosal speckling as well as duodenal/jejunal corrugations (Stander et al., 2010). Ultrasonographic view of a 2-month-old puppy suffering from CPV revealed generalized fluid distended, atonic small intestines with presumed normal intestinal wall thickness (Pennick et al., 1990).

As a result, ultrasonography is a non-invasive, fast and reliable method and may be employed as a helpful method for enteritis detection in order to exclude other intestinal diseases. In our first patient, intestinal wall thickening accompanied a view of intestinal loops that had lost their individualization (Figure 2). In addition to thickness, damaged wall layering seems to be an important finding which differentiates inflammatory diseases. Loss of layering has been reported in severe enteritis or in hemorrhagic infiltration. Two patterns of increased mucosal echogenicity reported are hyperechoic striations and hyperechoic speckles. Hyperechoic striations are specific for protein-losing enteropathy. Hyperechoic speckles are sensitive for determining the presence of inflammatory disease but they are non-specific for differentiating disease category and activity. Corrugation of the small bowel appears as regular waves of undulated bowel segments, a sensitive but nonspecific indicator of intestinal disorders and can be seen in association with parvoviral enteritis (Agut, 2009). In Dog-1, damaged wall layering was in concordance with severe enteritis and hemorrhagic infiltration view. In Dog-2 effected mucosal walls and bowel segment distension was related to the disease (Figure 4). Ultrasonographic examination of the gastrointestinal tract is often challenged by the presence of gas and/or faeces (Agut,
2009). Moreover, the gastrointestinal tract anatomy prevents continuous visualization from beginning to end. Yet, the investigation is commonly performed (Mantis, 2008) but lesion identification can be "hit or miss" due to the factors mentioned above. It is important to remember that not distinguishing all of the layers does not necessarily indicate pathology as gas artifact and limited resolution can lead to a false loss of the normal layering (Anderson, 2011). Since small intestinal wall thickness depends on the dog's weight, thicker walls should be viewed with suspicion. The appearance is not specific (Mantis, 2008). In some cases of gastrointestinal diseases, the utility of abdominal USG over the other diagnostic tests is debated. According to a recent study, in dogs with chronic vomiting, the contribution of abdominal USG was only in $27 \%$ of the cases (Benigni, 2012). In dogs evidence of loss of layering and mild to moderate intestinal wall thickening are excellent predictive factors in differentiating enteritis. Inflammatory lymph nodes can be enlarged but generally maintain their normal shape. Hemorrhage and necrosis can cause lymph-nodal mixed echogenicity and irregular outline (Benigni, 2012). In our case, USG of both patients' intestinal tract revealed loss of layering and generalized thickening of intestinal wall (Figure 3 and Figure 4). Ultrasonographic changes associated with CPV include fluid filled, atonic small and large intestines, duodenal and jejunal mucosal layer thinning with or without indistinct wall layers and irregular mucosal surfaces, duodenal/jejunal corrugations. Each of these changes cannot be considered as pathognomonic for CPV but if observed during abdominal pediatric USG, the clinician should be alerted of underlying CPV (Stander, 2009).

Despite it was hypothesized that acute CPV causes characteristic ultrasonographic gastrointestinal alterations, USG cannot be used to definitively diagnose CPV. Yet, daily ultrasonographic examination is helpful to observe the progression of the disease (Pennick et al., 1990). Moreover, though being debatable due to its particular significance by obtaining sharp images, USG provides confirmation of a gastrointestinal disease's diagnosis suspected on clinical signs (Malancus, 2010). It is concluded that intestinal USG may be helpful as an accessory diagnostic method in confirming CPV diagnosis apart from other primary clinical and hematological examinations especially in establishing a differential diagnosis. 


\section{References}

Agut, A., (2009). Ultrasonography of the small intestine in small animals. Veterinary Focus, 19(1), 20-28.

Anderson, K. L., (2011). Ultrasonography of the gastrointestinal tract,

Retrived from http://www.academicserver.cvm.umn.edu/ radiology/CVM6105/2011/Anderson/pdf/USofGI.pdf.

Benigni, L., (2012). Update on gastrointestinal imaging. Retriwed from http://web.aimgroupinternational.com/2012/ unigastro/wordpress/wpcontent/uploads/2012/05 Benigni_Livia2.pdf

Decaro, N., \& Buonavoglia, C. (2012). Canine parvovirus-a review of epidemiological and diagnostic aspects, with emphasis on type 2c. Veterinary Microbiology. 155(1), 1-12.

Goddard, A., \& Leisewitz, A. L. (2010). Canine parvovirus. Veterinary Clinics of North America Small Animal Practice, 40 (6), 1041-1053.

Malancuş, R. N, Tofan (Malancuş), C., \& Solcan, G. H. (2010). The use of ultrasonography in the diagnosis of gastrointestinal disease in dogs. Bulletin UASVM; Veterinary Medicine. 67(2), 143-149.

Mantis, P. (2008). Ultrasonography of the gastrointestinal tract and pancreas of the dog and cat.

Retriewed from http://www.sid.ir/en/VEWSSID/ J_pdf/11592008S07.pdf

Marcovich, J. E., Stucker, K. M., Carr, A. H., Harbison, C. E., Scarlett, J. M., \& Parrish, C. R. (2012). Effects of canine parvovirus strain variations on diagnostic test results and clinical management of enteritis in dogs. Journal of American Veterinary Medical Association, 241(1), 66-72.
Kumar, M., \& Nandi, S. (2010). Molecular typing of canine parvovirus variants by polymerase chain reaction and restriction enzyme analysis. Transboundary and Emerging Diseases, 57(6), 458-463.

Ntafis, V., Xylouri, E., Kalli, I., Desario, C., Mari, V., Decaro, N., \& Buonavoglia, C. (2010). Characterization of canine parvovirus type 2 (CPV-2) variants circulating in Greece. Journal of Veterinary Diagnostic Investigation, 22(5), 737-740.

Nwoha, R. I. O. (2011). Parvoviral Enteritis in a dog: Case report and rewiev of the literature. Continental Journal Veterinary Sciences, 5(1), 6-10.

Pennick, D. G, Nyland, T. G, Kerr, L. Y. \& Fisher, P. E. (1990). Ultrasonographic evaluation of gastrointestinal diseases in small animals. Veterinary Radiology, 31(3),134-141.

Prittie, J. (2004). Canine parvoviral enteritis: a review of diagnosis, management and prevention. Journal of Veterinary Emergency and Critical Care, 14(3), 167-176.

Sharma, A., Thompson, M. S., Scrivani, P. V., Dykes, N. L., Yeager, A. E., Freer, S. R., \& Erb, H. N. (2010). Comparison of radiography and ultrasonography for diagnosing small intestinal mechanical obstruction in vomiting dogs. Veterinary Radiology and Ultrasound, 52(3), 248-255.

Stander, N. (2009). The ultrasonographic appearance of the gastrontestinal tract in normal and parvoviral infected puppies. PhD Thesis, University of Pretoria. Pretoria, South Africa.

Stander, N, Wagner, W. M., Goddard, A. \& Kırberger, R. M. (2010). Ultrasonographic appearance of canine parvoviral enteritis in puppies. Veterinary Radiology and Ultrasound, 5 (1), 69-74 